\title{
Die Eisenbibliothek
}

\author{
Von Clemens Moser
}

Vor 40 Jahren, am 31. Dezember 1948, wurde die Eisenbibliothek - Stiftung der Georg Fischer AG - auf Initiative des damaligen Delegierten des Verwaltungsrates, Dr. Ernst Müller, gegründet. Die Stiftungsurkunde umschreibt den Zweck der Bibliothek kurz zusammengefaßt: «Erwerb, Sammlung und Betreuung von historischer und neuzeitlicher Eisenliteratur unter Einbezug ihrer Randgebiete. Kostenlose Benützung der Bibliothek für Wissenschaft und Technik, Förderung der Erforschung der Geschichte des Eisens.» 1952 konnte die Sammlung in den frisch renovierten Westflügel des ehemaligen Klarissenklosters Paradies, $4 \mathrm{~km}$ von Schaffhausen rheinaufwärts, verlegt werden. Die Räumlichkeiten können etwa 40000 Bücher aufnehmen. Neben den Magazinräumen stehen Lese- und Arbeitszimmer, Katalograum sowie Gästezimmer zur Nutzung zur Verfügung.

Gegenwärtig besitzt die Eisenbibliothek rund 33000 Einheiten. Unter diesen befinden sich zahlreiche Standardwerke der Eisenliteratur des 16. bis 19. Jahrhunderts, viele weniger bekannte Monographien und akademische Schriften, Regierungsverordnungen für das Eisenhüttengewerbe und den Bergbau. Das Schrifttum einiger Hilfswissenschaften und Nachbargebiete hat neben den rein naturwissenschaftlichen, eisen- und werksgeschichtlichen Büchern einen gebührenden Platz erhalten. Im Mittelpunkt der Nachbargebiete steht der Bergbau und die Lagerstättenkunde. Zum Schrifttum der Hilfswissenschaften gehören ausgewählte Werke über Geologie, Mineralogie sowie über die besonders wichtige Probierkunde, die heutige Metallurgie. Literatur aus dem Gebiet der reinen Naturwissenschaften wurde hauptsächlich aus der älteren Physik und Chemie in die Eisenbibliothek aufgenommen und dies besonders deshalb, weil die gewerbekundlichen, oder wie man auch sagen könnte, die technologischen Bücher früherer Jahrhunderte oft nicht von den rein naturwissenschaftlichen zu trennen sind, da sie inhaltlich ineinander übergehen; denn Wissenschaft und Gewerbe sind miteinander und aneinander emporgewachsen.

Prähistorische und metallgeschichtliche Werke, Bücher über Meteore, frühe Werke über angewandte Mechanik und Maschinen bilden neben dem Schrifttum über Waffen und Kunstschmiedearbeiten, den Eisenkunstguß und die Schlosserei einen spezifisch kulturgeschichtlichen Hintergrund der Eisenbibliothek. Werke über Eisenkonstruktionen, wie über eiserne Brükken, über die Verwendung von Eisen und Stahl beim Schiffsbau, bei Tief- 
und im Hochbau sind nach sorgfältiger Auswahl und Abgrenzung gegenüber der eigentlichen Literatur über Eisenverarbeitung ebenfalls in die Bibliothek aufgenommen worden und bilden einen natürlichen Rahmen.

Alte Reisebeschreibungen können kostbare Quellen für die Geschichte des Eisens und oft auch für seine Technologie sein, sie ergänzen eigentlich metallurgische Reisebeschreibungen in der Bibliothek.

\begin{abstract}
Der Sammlung berühmter Enzyklopädien oder enzyklopädischer Werke wurde eine besondere Aufmerksamkeit entgegengebracht. Sie sind Fixpunkte jeder Fachbibliothek, bilden die Enzyklopädien doch für bestimmte Zeitabschnitte gewissermaßen die Bilanz des jeweiligen gesamten Wissens, technischen und gewerblichen Könnens, sind wahre Gemälde des kulturellen und zivilisatorischen Fortschrittes. An dieser Stelle denkt man besonders an die Enzyklopädie von Diderot und d'Alembert, die Rechenschaft über Wissenschaft, Technik und Kunst zum Ausgang des 18. Jahrhunderts gibt, und an Johann Georg Krünitz' «Oekonomisch technologische Enzyklopädie» in 242 Bänden, deren Herausgabe erst 80 Jahre nach Erscheinen des ersten Bandes abgeschlossen war.

Die technische Zeitschrift, die sich ja in allen Kulturländern zu Ausgang des 18. Jahrhunderts selbständig machte und die sich im 19.Jahrhundert immer mehr spezialisierte, nimmt natürlich in der Eisenbibliothek einen breiten Raum ein. D.h. rund ein Drittel des Gesamtbestandes bilden diese Zeitschriften.
\end{abstract}

Dem alten schweizerischen Eisenbergbau, dem eisenerzeugenden Gewerbe und der Geschichte des Eisens in der Schweiz überhaupt wurde besondere Sorgfalt geschenkt. Der Standort «Helvetica» weist denn auch ein umfangreiches Schrifttum auf, ausgehend von der für die Geschichte des Eisens so wichtigen Latène-Zeit bis in die Gegenwart. Hier übernahm die Bibliothek auch archivalische Pflichten. Sämtliche Akten und Urkunden, die das Bergwerk am Gonzen betreffen, werden gesammelt, der Nachlaß einer großen schweizerischen Stahlhandlung aus Weinfelden behütet und seit kurzem das Archiv der Schweizerischen Stahlhandelszentrale betreut.

Nachdem der Bücherbestand der Eisenbibliothek in allgemeineren Zügen charakterisiert wurde, soll jetzt noch näher auf einige besonders wichtige Werke und Autoren zur Eisengeschichte hingewiesen werden.

Überblickt man die Eisenliteratur aller Jahrtausende, so findet man zunächst in der Antike nur verhältnismäßig wenige, und in zahlreichen Schriften verstreute Angaben über das Eisen und seine Herstellung. Insbesondere nach dem Zusammenbruch des Römischen Reiches sind die Spuren einer gewissen Leere gut zu erkennen. So konnten das ganze Mittelalter hindurch die Kenntnisse über das Eisen nicht sehr erweitert werden. Die Methoden des Eisengewerbes waren noch die alten geblieben - Überlieferun- 
gen aus der Welt der Antike, ein Erbe von Generation zu Generation. Was die Klöster als Hüter der abendländischen Kulturen erhalten konnten, ist auf dem Gebiete der Eisenliteratur außerst spärlich und stützt sich hauptsächlich auf vormittelalterliches Schrifttum.

Das älteste in der Sammlung befindliche Buch ist eine Handschrift von Albertus Magnus aus der ersten Hälfte des 13. Jahrhunderts mit dem Titel «De mineralibus libri quinque». Darin werden die Ansichten des gelehrten Kölner Dominikaners über das Wesen der Mineralien und Metalle überliefert, darunter auch über das Eisen. Das Mineralsystem übernahm er von Avicenna. Ein wichtiges Verdienst Albertus Magnus' war, das Wissen der damaligen Zeit zu sammeln und in etwa 70 Werken herauszugeben. Ein spätmittelalterlicher Chronist sagte über die wissenschaftliche Bedeutung des Albertus Magnus: «Wär er nit gewesen, Dutschelant wär ein Esel bliben».

Erst die Erfindung der Buchdruckerkunst wird zum Ansporn einer gesteigerten schriftstellerischen Tätigkeit auf allen Gebieten des Wissens. Auch die Literatur über Bergbau und Hüttenwesen nimmt nun, begünstigt durch die Regierungen und Fürsten, einen sichtbaren Aufschwung. Allerdings war das Eisen zu Anfang des 16. Jahrhunderts noch das Stiefkind unter den Metallen. Beschreibungen über seine Erschmelzung im Gegensatz zur Breite und Ausführlichkeit, mit denen andere Metalle beschrieben wurden, waren verhältnismäßig spärlich. Eisen wurde oft von Bauern im Nebengewerbe gewonnen; den Gelehrten schien es wegen der handwerklichen Einfachheit seiner Herstellung überhaupt nicht zu interessieren.

Von den sogenannten Frühdrucken oder Inkunabeln, die die Eisenbibliothek besitzt, soll nur kurz auf diejenige hingewiesen werden, die sich besonders mit der Naturgeschichte beschäftigt. Gemeint ist das Werk «De proprietatibus rerum» des irischen Wanderpredigers Bartholomaeus Anglicus, der im 13. Jahrhundert lebte. Sein Buch ist eine Art naturwissenschaftliche Enzyklopädie, mit einem Kapitel, das von Steinen und Metallen berichtet.

Georg Pawer, besser bekannt als Georgius Agricola, geb. am 24. März 1494 in Glauchau, gest. am 21. November 1555 in Chemnitz, war der wichtigste Erneuerer der technischen Wissenschaften in der ersten Hälfte des 16. Jahrhunderts. Nach seiner Tätigkeit als Lehrer in Zwickau begann Agricola 1522 das Medizinstudium an der Universität Leipzig. Etwa ein Jahr blieb er dort, um über Basel, wo er mit Erasmus von Rotterdam zusammentraf, nach Bologna zu fahren. Mit mehr als 90 Dozenten gehörte die dortige Universität zu einer der größten der damaligen Zeit. Neben seinen medizinischen 
Studien, so u. a. bei dem bekannten Chirurgen und Anatomen Berengario da Carpi, widmete sich Agricola noch hebräisch-arabischen Sprachstudien. 1524 erlangte er seine Doktorwürde und war im Herbst des gleichen Jahres in Venedig als Lektor der berühmten Verlagsfirma Aldus Manutius Nachf. tätig. Dort war er maßgebend für die Herausgabe der griechischen Textausgabe des Galen, die 1525 in fünf Bänden mit über 3000 Seiten erschien, verantwortlich und auch an der 1526 erschienenen Hippokrates-Ausgabe war er beteiligt.

Im gleichen Jahr zog Agricola wieder nach Deutschland, zuerst nach Zwickau, danach nach Chemnitz. Im Frühling 1527 ließ er sich in St. Joachimsthal nieder, wo er als Stadtarzt und -apotheker tätig war. Eineinhalb Jahre später erschien sein erstes bergbautechnisches Werk: Bermannus sive de re metallica dialogus (Bermannus oder ein Gespräch über den Bergbau). Die 1529 geschriebene Schrift erschien 1530 bei Froben in Basel, nachdem sie Erasmus befürwortet hatte. Das Empfehlungsschreiben dieses Gelehrten stellte eine seltene Auszeichnung dar, die Erasmus neben Agricola und Thomas Morus nur noch drei anderen Gelehrten zuteil werden ließ. Der «Bermannus» ist ein Lokalführer durch die Bergstadt Joachimsthal. Aufgrund dieser Schrift und seiner weiteren Werke wurde Agricola in neuerer Zeit als Begründer der Mineralogie, Metallurgie und Geologie gewürdigt. Die Bücher von Agricola bilden das Fundament für die Sammlungen der Eisenbibliothek.

Später schuf Agricola noch das mit 273 Holzschnitten bereicherte, meist technologische Werk «De Re Metallica Libri XII». Es stellt gewissermaßen die Krone seiner wissenschaftlichen Arbeiten dar, erschien aber erst wenige Monate nach seinem Tode im Frühling 1556. Die Absicht Agricolas, der «De Re Metallica» einen Abschnitt über die Eisengießerei einzufügen, konnte von ihm nicht mehr durchgeführt werden. Weitere Stellen über das Eisen muß man in den geologischen und mineralogischen Werken dieses Schriftstellers zusammensuchen. Faßt man sämtliche Stellen, die sich auf das Eisen beziehen, zusammen, so geben diese Bruchstücke doch noch ein recht gutes und deutliches Bild über den Stand des Eisenhüttenwesens des frühen 16. Jahrhunderts. Gesamthaft stellt die «De Re Metallica» das umfassendste Werk über Bergbau und Metallkunde des 16. Jahrhunderts dar.

Noch einige Jahre früher, im Jahre 1540, erschien die in toskanischer Sprache geschriebene «Della Pyrotechnia, Libri X» des Vanuccio Biringuccio. Es ist das erste eigentliche Handbuch der gesamten metallurgischchemischen Technologie. Der Rahmen ist enger gespannt, als derjenige der 
«De Re Metallica». Über das Eisen und seine Erze berichtet sie jedoch ausführlicher und enthält auch Angaben über die Eisengießerei. Die «De Re Metallica» und die «Della Pyrotechnia» ergänzen sich in idealer Weise; in ihnen wurde das Fundament für die spätere metallurgische Wissenschaft gelegt. Der Einfluß dieser beiden Bücher auf das technische Schrifttum erstreckt sich bis in das 18. Jahrhundert hinein. Kein Wunder also, daß die «De Re Metallica» in den Bibliotheken der größten Gelehrten des 16., 17. und 18. Jahrhunderts vertreten war, so auch in der Sammlung Isaak Newtons.

Zu den wichtigsten eisenkundlichen Büchern des 16. Jahrhunderts gehören natürlich auch die ersten Probierbücher. Diese beschäftigen sich vorwiegend mit dem Erschmelzen verschiedener Metalle, deren Veredelung und der daraus resultierenden Legierungen. Das berühmteste unter ihnen erschien 1574: «Beschreibung der allerfürnemisten mineralischen Ertzt und Bergwerksarten» von Lazarus Erker.

Der 1516 in Zürich geborene Arzt, Naturforscher und Philologe Conrad Gessner ist in bescheidener Auswahl seiner Werke auch in der Eisenbibliothek vertreten. Weil Gessner sich gleichermaßen als Arzt, Zoologe, Botaniker und Geologe hervortat, wurde er gelegentlich auch der deutsche Plinius genannt. Er gehört zu den deutschen Vätern der Botanik und neben Georgius Agricola auch zu den Mitbegründern der mineralogischen und geologischen Wissenschaft. Seine naturwissenschaftlichen Werke zeichnen sich durch ihre Sachlichkeit und wahrheitsgetreuen Naturbeschreibungen aus. Erkenntnistheoretische Spekulationen waren ihm fremd. Conrad Gessner überlieferte der Nachwelt eine Reihe fundamentaler philologischer Arbeiten, die heute als Grundlage für die vergleichende Sprachwissenschaft dienen. Unter seinen naturkundlichen Werken ist das in seinem Todesjahr 1565 in Zürich erschienene mineralogisch-geologische Werk «De omni rerum fossilium genere, gemmis, lapidibus, metallis, et huius modi, libri aliquo» besonders zu erwähnen. In dieser Schrift faßt Gessner neben eigenen Beiträgen einige besonders wichtige zeitgenössische und ältere Arbeiten anderer Wissenschaftler zusammen. Seine naturgeschichtlichen Werke wurden nicht zuletzt durch dieses Buch mit der großen Naturgeschichte des Cajus Secundus Plinius des Älteren, der «Historia naturalis s. historia mundi, libri XXXVII» verglichen, die in einer Ausgabe von 1519 auch in der Eisenbibliothek vertreten ist.

Elf Jahre nach Erscheinen von Agricolas «De Re metallica» wurde als eines der ersten Bücher über Berufskrankheiten Paracelsus' Werk "Von der Bergsucht und anderen Bergkrankheiten» publiziert. Auch dieser Autor 
konnte die Verbreitung seines Druckes nicht mehr erleben. Sein Werk wurde erst 1567 in Dillingen verlegt. Paracelsus erprobte vor allem die Heilwirkung mineralischer Substanzen und stieß dabei auf neue chemische Verbindungen. Er entwickelte an anorganischen Medikamenten hauptsächlich Antimon- und Kalium-Verbindungen.

Die Literatur des Eisenhüttenwesens und über das Eisengewerbe ist im 17. Jahrhundert spärlicher als im Vorausgegangenen. Dies ist leicht zu verstehen, denn das vor der Erfindung der Buchdruckerkunst gestaute Wissen fand während des 16. Jahrhunderts Zug um Zug seinen Weg in das gedruckte Buch. Neue Erkenntnisse mußten vorerst gesammelt werden. Aber auch die kulturzerstörenden Einflüsse des 30 jährigen Krieges machten sich innerhalb weiter Gebiete des Kontinents bemerkbar. Der Impuls aus der Richtung der wissenschaftlichen Forschung, welcher von den in der Mitte des 17. Jahrhunderts gegründeten Gelehrtengesellschaften in Frankreich, England und Deutschland ausging, wie von der französischen Akademie der Wissenschaften, der englischen Royal Society und den zahlreichen kleineren deutschen und anderen europäischen Akademien, vermochte die Technik und das Gewerbe erst im 18. Jahrhundert zu befruchten und war in der Eisenliteratur auch erst im Jahrhundert der Aufklärung spürbar. Auch seitens der Chemie ist während des 17. Jahrhunderts eine Befruchtung der Technologie des Eisens nicht festzustellen. Die Chemie hatte keine wesentlichen neuen Erkenntnisse zu verzeichnen, die führenden Chemiker steckten noch in der Alchemie und die positiven Ansätze Robert Boyles, die der Chemie eine neue Richtung wiesen, konnten sich noch weit in das 18. Jahrhundert hinein nicht auswirken. Praktiker, wie Dud Dudley und Sturtevant, setzten sich mit ihren der Zeit vorauseilenden Vorschlägen und Patenten, die Eisenerze, statt mit der teuren und oft nur schwer aufzutreibenden Holzkohle, mit mineralischer Kohle oder gar mit Torf zu verhütten, nicht durch.

Faßt man im 17. Jahrhundert die vielen lokalen Schriften der Eisenliteratur zusammen, sowie das ganze Schrifttum der Hilfswissenschaften, so ergibt sich trotzdem für die Eisenbibliothek eine ganz ansehnliche Sammlung.

Ein anderes Bild bietet das 18. Jahrhundert. Die Eisenliteratur nimmt, wie viele andere Zweige der Literatur im Jahrhundert der Aufklärung, einen ungeahnten Aufschwung. Jetzt beginnen die neuen Methoden der exakten Naturwissenschaften, der Chemie und Physik, das gelenkte Experiment, die Technik zu befruchten. 
Gleich einem Meteor am Himmel der Eisenliteratur erscheint im Jahre 1722 in Paris das von Antoine Ferchault de Réaumur geschriebene Werk: "L'art de convertir le fer forgé en acier et l'art d'aducir le fer fondu...» Eigentlich waren diese Memoiren dazu bestimmt, in einer von der französischen Akademie geplanten technischen Enzyklopädie zu erscheinen, zu deren Herausgeber Réaumur bestimmt wurde und an der er Zeit seines Lebens arbeitete. Diese Riesenaufgabe vermochte er nicht mehr zu bewältigen, hinterließ aber, als er 1757 starb, eine größere Anzahl Manuskripte. Unter Verwendung dieser Akten und als Gemeinschaftsarbeit zahlreicher Gelehrter, erschien das ein halbes Jahrhundert zuvor in Angriff genommene Werk schließlich unter dem Titel «Déscriptions des arts et métiers».

Die Réaumurschen Mémoiren können als Meilenstein der Eisenliteratur betrachtet werden. Es ist das erste metallurgische Werk der Neuzeit, in dem der gelenkte Laboratoriumsversuch und damit die moderne Forschungsmethode allein zur Geltung kommt. Es gibt hier keine Spekulationen, keine Wiederholung verstaubter Ideen aus der Gelehrtenstube des Mittelalters, der moderne Naturforscher löst ein Problem, welches ihm sein Land und sein Volk stellt, ein Problem der Technik, nur mit Methoden wie sie in der Physik seit Galilei, Hughens, und Newton selbstverständlich waren und wie sie nun mit diesem Werk von Réaumur auch Eingang in die Eisentechnik finden.

In Schweden, mit seinem Reichtum an Rasen- und Sumpferzen, aber auch an Erzlagerstätten größter Mächtigkeit und allererster Qualität, ist das Eisengewerbe seit Urzeiten beheimatet gewesen. Mit der Entdeckung der Lagerstätte in Dannemore zu Ausgang des 15. Jahrhunderts schwingt es sich zunächst auch, infolge seines Holzreichtums, zum wichtigsten eisenerzeugenden Land Europas auf. Das Haus Wasa erkannte die Bedeutung des Eisens für Land und Rüstung und unterstützte deshalb Bergbau und Hüttenwesen, Gelehrte und Techniker tatkräftig. Kein geringerer als der große nordische Philosoph und Naturforscher Emanuel Swedenborg, bereicherte im Jahre 1734 das Schrifttum über Eisen mit einem Werk, betitelt: "Regnum subterraneum sive minerale de Ferro» und schrieb damit das erste umfassendste Buch über die Eisenhüttenkunde. Swedenborg berichtet in aller Ausführlichkeit, wie das Eisen in Schweden hergestellt wurde und was er auf seinen Reisen im Ausland über die Eisengewinnung erfuhr.

Ein Versuch, die praktischen Kenntnisse, welche Réaumur und Swedenborg über die Eisenhüttenprozesse mitteilen, chemisch und physikalisch zu begründen, unternahm Sven Rinman mit seinem berühmt gewordenen Buch: «Försök till Järnets historia, med tillämping för Slöjder och Handtverk», 
welches 1781 in Stockholm gedruckt wurde und die erste systematische Eisenhüttenkunde darstellt. Wenige Jahre später, 1791, erschien ebenfalls in Stockholm die Schrift von Johen Carl Garney: «Handledning uti Svenska masmåsteriet». Diese behandelt den Hochofenbau und -betrieb ergänzt die Schriften und Bücher Rinmans.

Die bereits mit Réaumur erwähnte, von der französischen Akademie der Wissenschaften herausgegebene technische Enzyklopädie «Description des arts et métiers» nahm in französischer Übersetzung mit nur geringfügigen Kürzungen auch das Swedenborgsche Werk: «Regnum subteranneum...» als Artikel auf. Neben einigen Abschnitten über das Eisengewerbe, wie über die Schlosserei und die Messerschmiedekunst usw., enthält sie eine umfassende Arbeit des Marquis de Courtivron und Herrn Bouchu, betitelt: «Art des forges et fournaux à fer», dem jedoch sehr wahrscheinlich Manuskripte von Réaumur zugrunde lagen.

Die im Jahre 1774 erschienenen «Voyages métallurgiques» von G. Jars sind weltberühmt geworden; die auf Veranlassung der französischen Regierung unternommenen Reisen sind ein Beispiel fruchtbarer Zusammenarbeit zwischen Forschung und Staat geworden.

Erwähnt soll noch das große Werk das französischen Eisenhüttenmannes J.H. Hassenfratz von 1812 werden: «La sidérotechnie». Dieses Werk kann als Abschluß der Beschreibung der technischen Forschung betrachtet werden, die mit Réaumur und Swedenborg begann, mit dem oben erwähnten Rinmanschen Werke eine erste systematische Zusammenfassung fand und nun Hand in Hand mit der chemischen Forschung während des letzten Viertels des 18. Jahrhunderts wichtigste Fragen der Eisenhüttenkunde abklären konnte.

Nachdem ein Querschnitt durch die in der Eisenbibliothek vertretenen wichtigsten Werke und Schriften früherer Jahrhunderte gegeben wurde, müßte man sich eigentlich in derselben Ausführlichkeit der Literatur des 19. Jahrhunderts zuwenden, wenn nicht die Fülle der Bücher dieser Zeit den Rahmen dieses Artikel sprengen würden.

Die vorangehenden Erläuterungen zum Bücherbestand, die sich nur mit der alten Literatur befassen, könnten nun durch eine literaturgeschichtliche Beschreibung «neuerer» Bücher ergänzt werden. Doch hat die Bibliothek insbesondere Dank der Sammlung alter, historischer Werke weltweite Anerkennung gefunden.

Die Eisenbibliothek ist nach dem Willen der Stifterin zur freien Benutzung und Nutzung bestimmt. Die Sammlung bietet individuell angepaßte 
Öffnungszeiten - nach vorgängiger Anmeldung; wissenschaftliche Beratung bei Studienarbeit durch Fachkräfte und die Möglichkeit, bei längerem Studienaufenthalt im Klostergut untergebracht zu werden.

\section{Summary}

The Iron Library located in the Paradise convent is a foundation of Georg Fischer Ltd., Schaffhausen. The decisive impulse for its establishment in 1948 was given by Dr.h.c. Ernst Müller, managing director of the company for many years.

A comprehensive collection of books and documents was built up in course of time, their subject: iron and everything connected with it: history, mining, transformation, and use in crafts, industry and the arts. The Iron Library does not however limit itself to strict "iron litterature". It also collects works on related subjects: history of technology, metallurgy, old mining law etc. Today, the Iron Library comprises over 35000 volumes of all periods, stored for easy access acording to specific fields of knowledge.

In keeping with the founder's conception, the library is intended for unrestricted access and use by researchers and students of all fields. "Viris ferrum donatibus": To the men who gave us iron, is the motto of the library inscribed on the transom of the entrance door.

Clemens Moser

Eisenbibliothek

Klostergut Paradies

CH-8246 Langwiesen 